\title{
INTEGRATION IN A DYNAMICAL STOCHASTIC GEOMETRIC FRAMEWORK
}

\author{
Giacomo Aletti $^{1}$, Enea G. Bongiorno ${ }^{1}$ and Vincenzo Capasso ${ }^{1}$
}

\begin{abstract}
Motivated by the well-posedness of birth-and-growth processes, a stochastic geometric differential equation and, hence, a stochastic geometric dynamical system are proposed. In fact, a birth-and-growth process can be rigorously modeled as a suitable combination, involving the Minkowski sum and the Aumann integral, of two very general set-valued processes representing nucleation and growth dynamics, respectively. The simplicity of the proposed geometric approach allows to avoid problems of boundary regularities arising from an analytical definition of the front growth. In this framework, growth is generally anisotropic and, according to a mesoscale point of view, is non local, i.e. at a fixed time instant, growth is the same at each point of the space.
\end{abstract}

Mathematics Subject Classification. 60D05, 53C65, 60G20.

Received July 14, 2009. Revised November 26, 2009 and February 25, 2010.

\section{INTRODUCTION}

The importance of nucleation and growth processes is well known. They arise in several natural and technological applications ( $c f .[9,10]$ and references therein) such as, for example, solidification and phase-transition of materials, semiconductor crystal growth, biomineralization, and DNA replication, e.g. [22]. During the years, several authors studied stochastic spatial processes ( $c f .[16,28,36]$ and references therein) nevertheless they have essentially considered static approaches modeling real phenomena. For what concerns the dynamical point of view, a parametric birth-and-growth process was studied in [30,31]. A birth-and-growth process is a random closed sets (RaCS) family given by $\Theta_{t}=\bigcup_{n: T_{n}<t} \Theta_{T_{n}}^{t}\left(X_{n}\right)$, for $t \geq 0$, where $\Theta_{T_{n}}^{t}\left(X_{n}\right)$ is the RaCS obtained as the evolution up to time $t>T_{n}$ of the germ born at (random) time $T_{n}$ in (random) location $X_{n}$, according to some growth model. Analytical approaches are often used to study the propagation fronts of such processes. For example, in the level set theory, the front is moved by solving a Hamilton-Jacobi type equation written for a function the propagation front of which is a particular level set. In this framework, the well posedness of the initial value problem requires different smoothness conditions on the Hamilton-Jacobi equation and on the initial value (see at e.g. $[5,6]$ ). In some sense, regularity assumptions are due to the fact that growth is driven by a non negative normal velocity, i.e. at every instant $t$, a boundary point of the crystal $x \in \partial \Theta_{t}$ "grows" along the exterior unit normal vector, e.g. $[3,7,8,14,20]$. Hence, the existence of the exterior normal vector imposes regularity conditions on the growth front $\partial \Theta_{t}$. Nucleation process must be regular enough, usually

\footnotetext{
Keywords and phrases. Random closed set, Stochastic geometry, Birth-and-growth process, Set-valued process, Aumann integral, Minkowski sum.

${ }^{1}$ Department of Mathematics, University of Milan, via Saldini 50, 10133 Milan Italy. giacomo.aletti@unimi.it; enea.bongiorno@unimi.it; vincenzo.capasso@unimi.it
} 
spherical nucleus of infinitesimal radius is requested (nucleus can not be a point). Different parametric and non parametric estimations are proposed over the years, $c f .[2,9,12,15,19,29,32]$ and references therein.

This paper is an attempt to offer an original alternative approach based on a purely stochastic geometric point of view, in order to avoid regularity assumptions on the growth front describing birth-and-growth processes. In particular, we model the time evolution of a birth-and-growth process as a geometric stochastic differential equation of the following form

$$
\mathrm{d} \Theta_{t}=\oplus G_{t} \mathrm{~d} t \cup \mathrm{d} B_{t} \quad \text { or } \quad \Theta_{t+\mathrm{d} t}=\left(\Theta_{t} \oplus G_{t} \mathrm{~d} t\right) \cup \mathrm{d} B_{t}
$$

where $\left\{B_{t}\right\}_{t \in\left[t_{0}, T\right]}$ and $\left\{G_{t}\right\}_{t \in\left[t_{0}, T\right]}$ are an increasing closed set-valued process and a bounded convex closed set-valued process representing nucleation and growth, respectively. Roughly speaking, the increment $\mathrm{d} \Theta_{t}$, during an infinitesimal time interval $(t, t+\mathrm{d} t]$, is an enlargement due to an infinitesimal Minkowski addend $G_{t} \mathrm{~d} t$ and by the union of an infinitesimal nucleation $\mathrm{d} B_{t}$. As expected, the differential equation (1.1) has to be understood in integral form

$$
\Theta_{t}=\left(\Theta_{t_{0}} \oplus \int_{t_{0}}^{t} G_{\tau} \mathrm{d} \tau\right) \cup \bigcup_{s \in\left[t_{0}, t\right]}\left(\mathrm{d} B_{s} \oplus \int_{s}^{t} G_{\tau} \mathrm{d} \tau\right)
$$

so that the scope of this paper is to provide a rigorous mathematical meaning to (1.2). Clearly, these differential and integral equations allow us to handle a continuous time stochastic geometric dynamical system. Moreover, we deal with a non-local growth; i.e. growth is the same Minkowski addend at every $x \in \Theta_{t}$. Nevertheless, under a mesoscale hypothesis we can only consider constant growth region as described, for example, in [8]. Note that anisotropy growth occurs when $G_{t}$ is not a ball; different growths may be observed along different directions.

We want to observe that, the Minkowski sum was already employed in [27] to describe self-similar growth of a single convex germ.

In view of applications, in [1], the authors showed how the model leads to different and significant statistical results. In particular, they introduce different set-valued parametric estimators of the rate of growth of the process, that arise naturally from a decomposition via Minkowski sum and that are consistent as the observation window expands to the whole space. Moreover, keeping in mind that distributions of random closed sets are determined by hitting functionals and that the nucleation process cannot be observed directly, in [1], the authors provide an estimation procedure of the hitting function of the nucleation process.

The article is organized as follows. Section 2 contains some assumptions about (random) closed sets and their properties. For the sake of simplicity, we present, in Section 3, main results of the paper (that imply well-posedness of the model), whilst correspondent proofs are in Appendix A. Section 4 proposes some discussions and interpretations.

\section{Preliminary Results}

Let $\mathbb{N}, \mathbb{Z}, \mathbb{R}, \mathbb{R}_{+}$be the sets of all non-negative integer, integer, real and non-negative real numbers respectively. Let $\mathfrak{X}, \mathfrak{X}^{*}, B_{1}^{*}$ be a Banach space, its dual space and the unit ball of the dual space centered in the origin respectively. We shall consider

$$
\begin{aligned}
& \mathfrak{P}=\text { the family of all subsets of } \mathfrak{X}, \quad \mathfrak{P}^{\prime}=\mathfrak{P} \backslash\{\emptyset\} \\
& \mathbb{F}=\text { the family of all closed subsets of } \mathfrak{X}, \quad \mathbb{F}^{\prime}=\mathbb{F} \backslash\{\emptyset\} \text {. }
\end{aligned}
$$

The subscripts $b, k$ and $c$ denote boundedness, compactness and convexity properties respectively (e.g. $\mathbb{F}_{k c}$ denotes the family of all compact convex subsets of $\mathfrak{X}$ ). 
For all $A, B \subseteq \mathfrak{X}$ and $\alpha \in \mathbb{R}_{+}$, we define

$$
\begin{aligned}
A+B & =\{a+b: a \in A, b \in B\}=\bigcup_{b \in B} b+A, & & \text { (Minkowski sum) } \\
\alpha \cdot A & =\alpha A=\{\alpha a: a \in A\}, & & \text { (Scalar product) }
\end{aligned}
$$

By definition, for any $A \subseteq \mathfrak{X}, \alpha \in \mathbb{R}_{+}$, we have $\emptyset+A=\emptyset=\alpha \emptyset$. It is well known that + is a commutative and associative operation with a neutral element but $\left(\mathfrak{P}^{\prime},+\right)$ is not a group $(c f$. [33]). The following relations are useful in the sequel (see [34]): for all $A, B, C \subseteq \mathfrak{X}$

$$
\begin{gathered}
(A \cup B)+C=(A+C) \cup(B+C) \\
\text { if } B \subseteq C, \quad A+B \subseteq A+C .
\end{gathered}
$$

In the following, we shall work with closed sets. In general, if $A, B \in \mathbb{F}^{\prime}$ then $A+B$ does not belong to $\mathbb{F}^{\prime}$ (e.g., in $\mathfrak{X}=\mathbb{R}$ let $A=\{n+1 / n: n>1\}$ and $B=\mathbb{Z}$, then $\{1 / n=(n+1 / n)+(-n)\} \subset A+B$ and $1 / n \downarrow 0$, but $0 \notin A+B)$. In view of this fact, we define $A \oplus B=\overline{A+B}$ where $\overline{(\cdot)}$ denotes the closure in $\mathfrak{X}$.

For any $A, B \in \mathbb{F}^{\prime}$ the Hausdorff distance (or metric) is defined by

$$
\delta_{H}(A, B)=\max \left\{\sup _{a \in A} \mathrm{~d}(a, B), \sup _{b \in B} \mathrm{~d}(b, A)\right\}, \quad \text { where } \quad \mathrm{d}(a, B)=\inf _{b \in B}\|a-b\|_{\mathfrak{X}} .
$$

For all $\left(x^{*}, A\right) \in B_{1}^{*} \times \mathbb{F}^{\prime}$, the support function is defined by $s\left(x^{*}, A\right)=\sup _{a \in A} x^{*}(a)$. It can be proved $(c f .[4,21])$ that for each $A, B \in \mathbb{F}_{b c}^{\prime}$,

$$
\delta_{H}(A, B)=\sup \left\{\left|s\left(x^{*}, A\right)-s\left(x^{*}, B\right)\right|: x^{*} \in B_{1}^{*}\right\} .
$$

Let $(\Omega, \mathfrak{F})$ be a measurable space with $\mathfrak{F}$ complete with respect to some $\sigma$-finite measure, let $X: \Omega \rightarrow \mathfrak{P}$ be a set-valued map, and

$$
\begin{aligned}
D(X) & =\{\omega \in \Omega: X(\omega) \neq \emptyset\} & \text { be the domain of } X, \\
X^{-1}(A) & =\{\omega \in \Omega: X(\omega) \cap A \neq \emptyset\}, \quad A \subset \mathfrak{X}, & \text { be the inverse image of } X .
\end{aligned}
$$

Roughly speaking, $X^{-1}(A)$ is the set of all $\omega$ such that $X(\omega)$ hits set $A$. Different definitions of measurability for set-valued functions are developed over the years by several authors ( $c f .[4,13,23,24]$ and reference therein). Here, we'll use the following facts.

Definition 2.1. $X$ is measurable if, for each $O$, open subset of $\mathfrak{X}, X^{-1}(O) \in \mathfrak{F}$.

Proposition 2.2 (See $[24]$ ). $X: \Omega \rightarrow \mathfrak{P}$ is a measurable set-valued map if and only if $D(X) \in \mathfrak{F}$, and

$$
\begin{array}{cl}
\left(D(X), \mathfrak{F}^{\prime}\right) & \rightarrow\left(\mathbb{R}, \mathcal{B}_{\mathbb{R}}\right) \\
\omega & \mapsto \mathrm{d}(x, X(\omega))
\end{array}
$$

is a measurable function of $\omega \in D(X)$ for each $x \in \mathfrak{X}$, where $\mathfrak{F}^{\prime}=\{A \cap D(X): A \in \mathfrak{F}\}$.

Let $\mu$ be a positive measure on $(\Omega, \mathfrak{F})$, then, from now on, $\mathcal{U}\left[\Omega, \mathfrak{F}, \mu ; \mathbb{F}^{\prime}\right]\left(=\mathcal{U}\left[\Omega ; \mathbb{F}^{\prime}\right]\right.$ if no ambiguity may arise) denotes the family of $\mathbb{F}^{\prime}$-valued measurable maps (analogous notation holds whenever $\mathbb{F}^{\prime}$ is replaced by another family of subsets of $\mathfrak{X})$. Let $(\Omega, \mathfrak{F}, \mathbb{P})$ be a complete probability space. A RaCS $X$ is an element of $\mathcal{U}\left[\Omega, \mathfrak{F}, \mathbb{P} ; \mathbb{F}^{\prime}\right]$. It can be proved (see [25]) that, if $X, X_{1}, X_{2}$ are RaCS and if $\xi$ is a measurable real-valued function, then $X_{1} \oplus X_{2}, X_{1} \ominus X_{2}, \xi X$ and (Int $\left.X\right)^{C}$ are RaCS. Moreover, if $\left\{X_{n}\right\}_{n \in \mathbb{N}}$ is a sequence of RaCS then $X=\overline{\bigcup_{n \in \mathbb{N}} X_{n}}$ is a $\operatorname{RaCS}$, too.

Let $X$ be a RaCS, then $T_{X}(K)=\mathbb{P}(X \cap K \neq \emptyset)$, for all $K \in \mathbb{F}_{k}$, is its hitting function (or Choquet capacity functional). The well known Choquet-Kendall-Matheron Theorem states that, the probability law $\mathbb{P}_{X}$ of any RaCS $X$ is uniquely determined by its hitting function (see [26]) and hence by $Q_{X}(K)=1-T_{X}(K)$. 
Let $(\Omega, \mathfrak{F}, \mu)$ be a finite measure space (although most of the results hold for $\sigma$-finite measures space). The Aumann integral of $X \in \mathcal{U}\left[\Omega, \mathfrak{F}, \mu ; \mathbb{F}^{\prime}\right]$ is defined by

$$
\int_{\Omega} X \mathrm{~d} \mu=\left\{\int_{\Omega} x \mathrm{~d} \mu: x \in S_{X}\right\},
$$

where $S_{X}=\left\{x \in L^{1}[\Omega ; \mathfrak{X}]: x \in X \mu\right.$-a.e. $\}$ and $\int_{\Omega} x \mathrm{~d} \mu$ is the usual Bochner integral in $L^{1}[\Omega ; \mathfrak{X}]$. Moreover, $\int_{A} X \mathrm{~d} \mu=\left\{\int_{A} x \mathrm{~d} \mu: x \in S_{X}\right\}$ for $A \in \mathfrak{F}$. If $\mu$ is a probability measure, we denote the Aumann integral by $\mathbb{E} X=\int_{\Omega} X \mathrm{~d} \mu$.

One may define $\int_{\Omega} X \mathrm{~d} \mu$ as a limit of integrals of simple RaCS, see [25], Def. 2.2.4 (Bochner integral)). The two definitions are equivalent in our framework (see ([25], Thm. 2.2.5)). We use the definition of Aumann integral in the proof of Proposition 3.7.

Let $X \in \mathcal{U}\left[\Omega, \mathfrak{F}, \mu ; \mathbb{F}^{\prime}\right]$, it is integrably bounded, and we shall write $X \in L^{1}\left[\Omega, \mathfrak{F}, \mu ; \mathbb{F}^{\prime}\right]=L^{1}\left[\Omega ; \mathbb{F}^{\prime}\right]$, if $\delta_{H}(X,\{0\}) \in L^{1}[\Omega, \mathfrak{F}, \mu ; \mathbb{R}]$.

\section{Geometric RAndom process}

Let us recall that the main purpose of this paper is the well-posedness of

$$
\Theta_{t}=\left(\Theta_{t_{0}} \oplus \int_{t_{0}}^{t} G_{\tau} \mathrm{d} \tau\right) \cup \bigcup_{s \in\left[t_{0}, t\right]}\left(\mathrm{d} B_{s} \oplus \int_{s}^{t} G_{\tau} \mathrm{d} \tau\right)
$$

and hence the existence of such a random "geometric integral". In other words, under what conditions is $\Theta_{t}$ a RaCS? It is well known that finite union and Minkowski addition of RaCS are RaCS too. Thus, this problem can be splitted in, essentially, two questions. Is $\int_{s}^{t} G_{\tau} \mathrm{d} \tau$ a RaCS? How can we handle the uncountable unions of RaCS in (1.2)? The answers will be given in the Section 3.2, based on the following assumptions.

\subsection{Model assumptions}

From now on, let us consider the following assumptions.

(A-0) $\quad-\left(\mathfrak{X},\|\cdot\|_{\mathfrak{X}}\right)$ is a reflexive Banach space with separable dual space $\left(\mathfrak{X}^{*},\|\cdot\|_{\mathfrak{X}^{*}}\right)$, (then, $\mathfrak{X}$ is separable too, see ([18], Lem. II.3.16 p. 65)).

$-\left[t_{0}, T\right] \subset \mathbb{R}$ is the time observation interval (or time interval),

- $\left(\Omega, \mathfrak{F},\left\{\mathfrak{F}_{t}\right\}_{t \in\left[t_{0}, T\right]}, \mathbb{P}\right)$ is a filtered probability space, where the filtration $\left\{\mathfrak{F}_{t}\right\}_{t \in\left[t_{0}, T\right]}$ is assumed to have the usual properties.

(Nucleation Process). $B=\left\{B(\omega, t)=B_{t}: \omega \in \Omega, t \in\left[t_{0}, T\right]\right\}$ is a process with non-empty closed values, i.e. $B: \Omega \times\left[t_{0}, T\right] \rightarrow \mathbb{F}^{\prime}$, such that

(A-1) $B_{t_{0}}=\Theta_{t_{0}}$ and $B(\cdot, t) \in \mathcal{U}\left[\Omega, \mathfrak{F}_{t}, \mathbb{P} ; \mathbb{F}^{\prime}\right]$, for every $t \in\left[t_{0}, T\right]$; i.e. $B_{t}$ is an adapted (to $\left\{\mathfrak{F}_{t}\right\}_{t \in\left[t_{0}, T\right]}$ ) process. (A-2) $B_{t}$ is increasing: for every $t, s \in\left[t_{0}, T\right]$ with $s<t, B_{s} \subseteq B_{t}$.

Roughly speaking, $B_{t}$ collects all nucleations up to time $t$.

(Growth Process). $G=\left\{G_{t}=G(\omega, t): \omega \in \Omega, t \in\left[t_{0}, T\right]\right\}$ is a process with non-empty closed values, i.e. $G: \Omega \times\left[t_{0}, T\right] \rightarrow \mathbb{F}^{\prime}$, such that

(A-3) for every $\omega \in \Omega$ and $t \in\left[t_{0}, T\right], 0 \in G(\omega, t)$.

(A-4) for every $\omega \in \Omega$ and $t \in\left[t_{0}, T\right], G(\omega, t)$ is convex, i.e. $G: \Omega \times\left[t_{0}, T\right] \rightarrow \mathbb{F}_{c}^{\prime}$.

(A-5) there exists $K \in \mathbb{F}_{b}^{\prime}$ such that $G(\omega, t) \subseteq K$ for every $t \in\left[t_{0}, T\right]$ and $\omega \in \Omega$.

Remark 3.1. We note that no assumptions are made on the regularity of the boundary of nucleation process and, hence, on the initial value $B_{t_{0}}=\Theta_{t_{0}}$, so that point processes are acceptable nucleations. Smoothness conditions are indeed necessary using the analytical approach of level set theory (see e.g. [5]). 
For what concerns Assumption (A-5), $G(\omega, t) \in \mathbb{F}_{b}^{\prime}$ and $\delta_{H}(G(\omega, t),\{0\}) \leq \delta_{H}(K,\{0\})$, for any $(\omega, t) \in$ $\Omega \times\left[t_{0}, T\right]$. This upper bound on $G$ is reasonable for most practical applications, since $G$ represents the growth speed of crystal, which usually remains finite.

Further, convexity hypothesis (A-4) is not so restrictive. In fact, since the Lebesgue measure is atomless, it can be proved (see ([25], Cor. 2.1.6)) that, whenever integrals exist, the following expression holds

$$
\overline{\int_{a}^{b} G(\omega, \tau) \mathrm{d} \tau}=\overline{\int_{a}^{b} \overline{c o} G(\omega, \tau)} \mathrm{d} \tau .
$$

In other words, convexity of $G$ is not a prerequisite for the convexity of its Lebesgue integral. Thus, whenever (1.2) is well posed, $\Theta$ will be the same employing a non-convex process $G$ or employing its convex hull $(\overline{c o} G)$.

Finally, convexity of $G$ does not imply convexity of $\Theta$, since $B$ is not, in general, a convex set.

In order to establish the well-posedness of the integral $\int_{t_{0}}^{t} G_{s} \mathrm{~d} s$ in (1.2), let us consider a suitable hypothesis of measurability for $G$. A $\mathbb{F}^{\prime}$-valued process $G=\left\{G_{t}\right\}_{t \in\left[t_{0}, T\right]}$ has left continuous trajectories on $\left[t_{0}, T\right]$ if, for every $\bar{t} \in\left[t_{0}, T\right]$ with $t<\bar{t}$,

$$
\lim _{t \rightarrow \bar{t}} \delta_{H}(G(\omega, t), G(\omega, \bar{t}))=0, \quad \text { a.s. }
$$

The $\sigma$-algebra on $\Omega \times\left[t_{0}, T\right]$ generated by the processes $\left\{G_{t}\right\}_{t \in\left[t_{0}, T\right]}$ with left continuous trajectories on $\left[t_{0}, T\right]$, is called the previsible (or predictable) $\sigma$-algebra and it is denoted by $\mathcal{P}$.

Proposition 3.2. The previsible $\sigma$-algebra is also generated by the collection of random sets $A \times\left\{t_{0}\right\}$ where $A \in \mathfrak{F}_{t_{0}}$ and $A \times(s, t]$ where $A \in \mathfrak{F}_{s}$ and $(s, t] \subset\left[t_{0}, T\right]$.

Then let us consider the following assumption.

(A-6) $G$ is $\mathcal{P}$-measurable.

\subsection{Main results}

For the sake of simplicity, let us present the main results which proofs will be given in Section A. Let us assume conditions from (A-0) to (A-6). For every $t \in\left[t_{0}, T\right] \subset \mathbb{R}, n \in \mathbb{N}$ and $\Pi=\left(t_{i}\right)_{i=0}^{n}$ partition of $\left[t_{0}, t\right]$, let us define

$$
\begin{aligned}
& s_{\Pi}(t)=\left(B_{t_{0}} \oplus \int_{t_{0}}^{t} G(\tau) \mathrm{d} \tau\right) \cup \bigcup_{i=1}^{n}\left(\Delta B_{t_{i}} \oplus \int_{t_{i}}^{t} G(\tau) \mathrm{d} \tau\right) \\
& S_{\Pi}(t)=\left(B_{t_{0}} \oplus \int_{t_{0}}^{t} G(\tau) \mathrm{d} \tau\right) \cup \bigcup_{i=1}^{n}\left(\Delta B_{t_{i}} \oplus \int_{t_{i-1}}^{t} G(\tau) \mathrm{d} \tau\right)
\end{aligned}
$$

where $\Delta B_{t_{i}}=B_{t_{i}} \backslash B_{t_{i-1}}^{o}\left(B_{t_{i-1}}^{o}\right.$ denotes the interior set of $\left.B_{t_{i-1}}\right)$ and where the integral is in the Aumann sense with respect to the Lebesgue measure $\mathrm{d} \tau=\mathrm{d} \mu_{\lambda}$. We write $s_{\Pi}$ and $S_{\Pi}$ instead of $s_{\Pi}(t)$ and $S_{\Pi}(t)$ when the dependence on $t$ is clear.

Proposition 3.3 collects some measurability and integrability properties of growth process; in particular, it shows that $\int_{a}^{b} G(\cdot, \tau) \mathrm{d} \tau$ is a RaCS with non-empty bounded convex values. Then, Proposition 3.4 guarantees that both $s_{\Pi}$ and $S_{\Pi}$ are well defined RaCS, further, Proposition 3.5 shows $s_{\Pi} \subseteq S_{\Pi}$ as a consequence of different time intervals integration: if the time interval integration of $G$ increases then the integral of $G$ does not decrease with respect to set-inclusion (Lem. A.3). Proposition 3.6 means that $\left\{s_{\Pi}\right\}\left(\left\{S_{\Pi}\right\}\right)$ increases (decreases) whenever a refinement of $\Pi$ is considered. At the same time, Proposition 3.7 implies that $s_{\Pi}$ and $S_{\Pi}$ become close to each other (in the Hausdorff distance sense) when partition $\Pi$ becomes finer. The "limit" is independent on the choice of the refinement as consequence of Proposition 3.8.

Corollary 3.9 means that, given any $\left\{\Pi_{j}\right\}_{j \in \mathbb{N}}$ refinement sequence of $\left[t_{0}, t\right]$, the random closed sets $s_{\Pi_{j}}$ and $S_{\Pi_{j}}$ play the same role that lower sums and upper sums have in classical analysis when we define the Riemann 
integral. In fact, if $\Theta_{t}$ denotes their limit value (see (3.3)), $s_{\Pi_{j}}$ and $S_{\Pi_{j}}$ are a lower and an upper approximation of $\Theta_{t}$ respectively. Note that, as a consequence of monotonicity of $s_{\Pi_{j}}$ and $S_{\Pi_{j}}$, we avoid problems that may arise considering uncountable unions in the integral expression in (1.2).

Proposition 3.3. Suppose (A-3), ., (A-6), and let $\mu_{\lambda}$ be the Lebesgue measure on $\left[t_{0}, T\right]$, then

- $G(\omega, \cdot) \in \mathcal{U}\left[\left[t_{0}, T\right], \mathcal{B}_{\left[t_{0}, T\right]}, \mu_{\lambda} ; \mathbb{F}_{b c}^{\prime}\right]$ for every $\omega \in \Omega$.

- $G(\cdot, t) \in \mathcal{U}\left[\Omega, \widetilde{\mathfrak{F}}_{t^{-}}, \mathbb{P} ; \mathbb{F}_{b c}^{\prime}\right]$ for each $t \in\left[t_{0}, T\right]$, where $\widetilde{\mathfrak{F}}_{t^{-}}$is the so called history $\sigma$-algebra i.e. $\widetilde{\mathfrak{F}}_{t^{-}}=$ $\sigma\left(\mathfrak{F}_{s}: 0 \leq s<t\right) \subseteq \mathfrak{F}$.

- $G \in L^{1}\left[\left[t_{0}, T\right], \mathcal{B}_{\left[t_{0}, T\right]}, \mu_{\lambda} ; \mathbb{F}_{b c}^{\prime}\right] \cap L^{1}\left[\Omega, \mathfrak{F}, \mathbb{P} ; \mathbb{F}_{b c}^{\prime}\right]$.

Furthermore, for every $a, b \in\left[t_{0}, T\right]$ and $\omega \in \Omega$, the integral $\int_{a}^{b} G(\omega, \tau) \mathrm{d} \tau$ is non-empty and the set-valued map

$$
\begin{aligned}
G_{a, b}: \Omega & \rightarrow \mathfrak{P}^{\prime} \\
\omega & \mapsto \int_{a}^{b} G(\omega, \tau) \mathrm{d} \tau
\end{aligned}
$$

is measurable, according to Definition 2.1. Moreover, $G_{a, b}$ is a non-empty, bounded convex RaCS.

Proposition 3.4. Let $\Pi$ be a partition of $\left[t_{0}, t\right]$. Both $s_{\Pi}$ and $S_{\Pi}$, defined in (3.1) and (3.2), are RaCS.

Proposition 3.5. Let $\Pi$ be a partition of $\left[t_{0}, t\right]$. Then $s_{\Pi} \subseteq S_{\Pi}$ almost surely.

Proposition 3.6. Let $\Pi$ and $\Pi^{\prime}$ be two partitions of $\left[t_{0}, t\right]$ such that $\Pi^{\prime}$ is a refinement of $\Pi$. Then, almost surely, $s_{\Pi} \subseteq s_{\Pi^{\prime}}$ and $S_{\Pi^{\prime}} \subseteq S_{\Pi}$.

Proposition 3.7. Let $\left\{\Pi_{j}\right\}_{j \in \mathbb{N}}$ be a refinement sequence of $\left[t_{0}, t\right]$ (i.e. $\left|\Pi_{j}\right| \rightarrow 0$ if $j \rightarrow \infty$ ). Then, almost surely, $\lim _{j \rightarrow \infty} \delta_{H}\left(s_{\Pi_{j}}, S_{\Pi_{j}}\right)=0$.

Proposition 3.8. Let $\left\{\Pi_{j}\right\}_{j \in \mathbb{N}}$ and $\left\{\Pi_{l}^{\prime}\right\}_{l \in \mathbb{N}}$ be two distinct refinement sequences of $\left[t_{0}, t\right]$, then, almost surely,

$$
\lim _{\substack{j \rightarrow \infty \\ l \rightarrow \infty}} \delta_{H}\left(s_{\Pi_{j}}, s_{\Pi_{l}^{\prime}}\right)=0 \quad \text { and } \quad \lim _{\substack{j \rightarrow \infty \\ l \rightarrow \infty}} \delta_{H}\left(S_{\Pi_{j}}, S_{\Pi_{l}^{\prime}}\right)=0 .
$$

Corollary 3.9. For every $\left\{\Pi_{j}\right\}_{j \in \mathbb{N}}$ refinement sequence of $\left[t_{0}, t\right]$, the following limits exist

$$
\left.\overline{\left(\bigcup_{j \in \mathbb{N}} s_{\Pi_{j}}\right.}\right), \overline{\left(\lim _{j \rightarrow \infty} s_{\Pi_{j}}\right)}, \lim _{j \rightarrow \infty} S_{\Pi_{j}}, \bigcap_{j \in \mathbb{N}} S_{\Pi_{j}},
$$

and they are equal almost surely. The convergence is taken with respect to the Hausdorff distance.

We are now ready to define the associated continuous time stochastic process.

Definition 3.10. Assume (A-0), .., (A-6). For every $t \in\left[t_{0}, T\right]$, let $\left\{\Pi_{j}\right\}_{j \in \mathbb{N}}$ be a refinement sequence of the time interval $\left[t_{0}, t\right]$ and let $\Theta_{t}$ be the RaCS defined by

$$
\left.\overline{\left(\bigcup_{j \in \mathbb{N}} s_{\Pi_{j}}(t)\right.}\right)=\overline{\left(\lim _{j \rightarrow \infty} s_{\Pi_{j}}(t)\right)}=\Theta_{t}=\lim _{j \rightarrow \infty} S_{\Pi_{j}}(t)=\bigcap_{j \in \mathbb{N}} S_{\Pi_{j}}(t),
$$

then, the family $\Theta=\left\{\Theta_{t}: t \in\left[t_{0}, T\right]\right\}$ is called geometric random process $G$-RaP (on $\left[t_{0}, T\right]$ ).

Theorem 3.11. Let $\Theta$ be a $G$-RaP on $\left[t_{0}, T\right]$, then $\Theta$ is a non-decreasing process with respect to the set inclusion, i.e.

$$
\mathbb{P}\left(\Theta_{s} \subseteq \Theta_{t}, \forall t_{0} \leq s<t \leq T\right)=1 .
$$

Moreover, $\Theta$ is adapted with respect to filtration $\left\{\mathfrak{F}_{t}\right\}_{t \in\left[t_{0}, T\right]}$. 


\section{Discussion}

In this section we want to stress out some characteristics of G-RaP.

A wide family of classical random sets and evolution processes can be represented by the previous model. In particular, the Boolean model (see, e.g., [16]) is a G-RaP with "null growth". Vice versa, it could be interesting to ask under which conditions the G-RaP $\Theta_{t}$ is a Boolean process for every $t$.

In the $\mathbb{R}^{d}$ case, one can ask if these results allow us to handle processes having Hausdorff dimension smaller than $d$. The answer is, in some sense, negative, since Minkowski sum is "fattening". For example, consider the $\mathbb{R}^{2}$ case, two 1-dimensional sets $A, B$ (two segments); then it is easy to see that, in general, $A \oplus B$ is a 2 -dimensional set (a parallelogram whose edges are the two segments). On the other hand, the growth process may be contained in a subspace of Hausdorff dimension smaller than $d$. As intersections and finite unions preserve RaCS, one may obtain fancy G-RaP with lower dimension.

In the following, we consider the problem of definition of a discrete time process and some statistical applications.

\subsection{Discrete time case and infinitesimal notations}

Here, we justify the infinitesimal notations pass through the definition of the discrete time process.

Let us consider $\Theta_{s}$ and $\Theta_{t}$ with $s<t$. Let $\left\{\Pi_{j}\right\}_{j \in \mathbb{N}}$ be a refinement sequence of $\left[t_{0}, t\right]$. For all $j \in \mathbb{N}$, let

$$
s_{\Pi_{j}}(s)=\left(B_{t_{0}} \oplus \int_{t_{0}}^{s} G(\tau) \mathrm{d} \tau\right) \cup \bigcup_{\substack{t^{\prime} \in \Pi_{j} \\ t^{\prime} \leq s}}\left(\Delta B_{t^{\prime}} \oplus \int_{t^{\prime}}^{s} G(\tau) \mathrm{d} \tau\right),
$$

then, it is easy to get

$$
s_{\Pi_{j}}(t)=\left(s_{\Pi_{j}}(s) \oplus \int_{s}^{t} G(\tau) \mathrm{d} \tau\right) \cup \bigcup_{\substack{t^{\prime} \in \Pi_{j} \\ t^{\prime}>s}}\left(\Delta B_{t^{\prime}} \oplus \int_{t^{\prime}}^{t} G(\tau) \mathrm{d} \tau\right) .
$$

Then, as a consequence of main results, whenever $\left|\Pi_{j}\right| \rightarrow 0$, we obtain

$$
\Theta_{t}=\left(\Theta_{s} \oplus \int_{s}^{t} G(\tau) \mathrm{d} \tau\right) \cup \lim _{\left|\Pi_{j}\right| \rightarrow 0} \bigcup_{\substack{t^{\prime} \in \Pi \\ t^{\prime}>s}}\left(\Delta B_{t^{\prime}} \oplus \int_{t^{\prime}}^{t} G(\tau) \mathrm{d} \tau\right) .
$$

The following notations

$$
G_{n}=\int_{s}^{t} G(\tau) \mathrm{d} \tau \quad \text { and } \quad B_{n}=\lim _{\left|\Pi_{j}\right| \rightarrow 0} \bigcup_{\substack{t^{\prime} \in \Pi \\ t^{\prime}>s}}\left(\Delta B_{t^{\prime}} \oplus \int_{t^{\prime}}^{t} G(\tau) \mathrm{d} \tau\right)
$$

lead us to the set-valued discrete time stochastic process

$$
\Theta_{n}= \begin{cases}\left(\Theta_{n-1} \oplus G_{n}\right) \cup B_{n}, & n \geq 1, \\ B_{0}, & n=0 .\end{cases}
$$

Note that, we can derive the discrete time process (4.2) directly by defining $\left\{B_{n}: n \geq 0\right\}$ and $\left\{G_{n}: n \geq 1\right\}$ as two families of RaCS, such that $B_{n}$ is $\mathfrak{F}_{n}$-measurable and $G_{n}$ is $\mathfrak{F}_{n-1}$-measurable, and where the filtration 
$\left\{\mathfrak{F}_{n}\right\}_{n \in \mathbb{N}}$ is assumed to have the usual properties. Thus, the discrete time process $\Theta=\left\{\Theta_{n}: n \geq 0\right\}$ is defined recursively by (4.2).

In view of (4.2), we are able to justify infinitesimal notations introduced in (1.1). In particular, from equation (4.1), whenever $\left|\Pi_{j}\right| \rightarrow 0$, we obtain

$$
\Theta_{t}=\left(B_{t_{0}} \oplus \int_{t_{0}}^{t} G(\tau) \mathrm{d} \tau\right) \cup \bigcup_{s=t_{0}}^{t}\left(\mathrm{~d} B_{s} \oplus \int_{s}^{t} G(\tau) \mathrm{d} \tau\right), \quad t \in\left[t_{0}, T\right] .
$$

Moreover, with a little abuse of this infinitesimal notation, we get two differential formulations

$$
\mathrm{d} \Theta_{t}=\oplus G_{t} \mathrm{~d} t \cup \mathrm{d} B_{t} \quad \text { and } \quad \Theta_{t+\mathrm{d} t}=\left(\Theta_{t} \oplus G_{t} \mathrm{~d} t\right) \cup \mathrm{d} B_{t} .
$$

\subsection{Statistical applications}

For the sake of completeness, we report on statistical results obtained in [1]. In fact, authors provided consistent estimators of the rate growth of $\Theta$ and the hitting function of $B_{n}$.

In view of applications, note that a sample of a birth-and-growth process is usually a time sequence of pictures that represent process $\Theta$ at different temporal step; so that (4.2) is a spontaneous way to modelize it. In particular, let us consider $\Theta_{n-1}, \Theta_{n}$ that, for the sake of simplicity, will be denoted in this section by $X$ and $Y$ respectively.

Note that, in practical cases, data are bounded by some observation window and, in order to reduce the arisen edge effects, the authors in [1] considered the following estimators of $G$.

$$
\begin{aligned}
\widehat{G}_{W}^{1} & =\left(Y_{W} \ominus \check{X}_{W \ominus \check{K}}\right) \cap K, \\
\widehat{G}_{W}^{2} & =\left(\left[Y_{W} \cup\left(\partial_{W}^{\oplus K} X_{W}\right)\right] \ominus \check{X}_{W}\right) \cap K ;
\end{aligned}
$$

where $\breve{X}=\{-x: x \in X\}$ is the symmetric set (with respect to the origin) of $X, W$ is the (bounded) observation window, in the right side subscript notation denotes intersection (for example, $Y_{W}=Y \cap W$ ), $\ominus$ denotes the Minkowski subtraction defined by $A \ominus B=\left(A^{C}+B\right)^{C}, K$ is a compact set such that $K \supseteq G=Y \ominus \check{X}$, and $\left(\partial_{W}^{\oplus K} X_{W}\right)=\overline{\left(X_{W}+K\right) \backslash W}$. Thus, as the standard statistical scheme for spatial processes suggests (see [28]), the authors proved that $\widehat{G}_{W}^{1}, \widehat{G}_{W}^{2}$ are consistent estimators of $G$ as the observation window expands to the whole space $W \uparrow \mathfrak{X}$. So, let us consider a convex averaging sequence of sets $\left\{W_{i}\right\}_{i \in \mathbb{N}}$ in $\mathfrak{X}$ [17], i.e. each $\left\{W_{i}\right\}$ is convex and compact, $W_{i} \subset W_{i+1}$ for all $i \in \mathbb{N}$ and

$$
\sup \left\{r \geq 0: B(x, r) \subset W_{i} \text { for some } x \in W_{i}\right\} \uparrow \infty, \text { as } i \rightarrow \infty .
$$

Proposition 4.1 (See [1]). Let $Y, X$ be RaCS, let $0 \in G=Y \ominus \check{X} \subseteq K$. Thus, for any $W_{1}, W_{2}$ with $W_{2} \supseteq W_{1}$, $G \subseteq \widehat{G}_{W_{2}}^{1} \subseteq \widehat{G}_{W_{1}}^{1}$. In particular, $\bigcap_{i \in \mathbb{N}} \widehat{G}_{W_{i}}^{1}=G$ and $\lim _{i \rightarrow \infty} \delta_{H}\left(\widehat{G}_{W_{i}}^{1}, G\right)=0$ (i.e. $\widehat{G}_{W}^{1}$ is consistent).

Moreover, for every $W \in \mathbb{F}^{\prime}$, it holds $G \subseteq \widehat{G}_{W}^{2} \subseteq \widehat{G}_{W}^{1}$. As a consequence, $\widehat{G}_{W}^{2}$ is consistent too (i.e. if $W \uparrow \mathfrak{X}$, then $\widehat{G}_{W}^{2} \downarrow G$ ).

Figure 1 is a computational application of above proposition.

From the birth-and-growth process point of view, it is also interesting to test whenever the nucleation process $B=\left\{B_{n}\right\}_{n \in \mathbb{N}}$ is a specific RaCS (for example a Boolean model or a point process). In general, the $n$th nucleation $B_{n}$ can not be directly observed, since it can be overlapped by other nuclei or by their evolutions. Nevertheless, in [1], authors provided consistent estimators of the hitting function $T_{B_{n}}(\cdot)$ associated to the nucleation process.

A regular closed set in $\mathfrak{X}$ is a closed set $X \in \mathbb{F}^{\prime}$ for which $X=\overline{\operatorname{Int} X}$. For any $K \in \mathbb{F}_{k}$, let $\widetilde{Q}_{B, W}(K)=$ $\widehat{Q}_{Y, W}(K) / \widehat{Q}_{X+\widehat{G}_{W}, W}(K)$, where $\widehat{Q}_{(\cdot)}=1-\widehat{T}_{(\cdot)}$ is defined in [28] and $\widehat{G}_{W}$ is one between $\widehat{G}_{W}^{2}$ and $\widehat{G}_{W}^{1}$. 

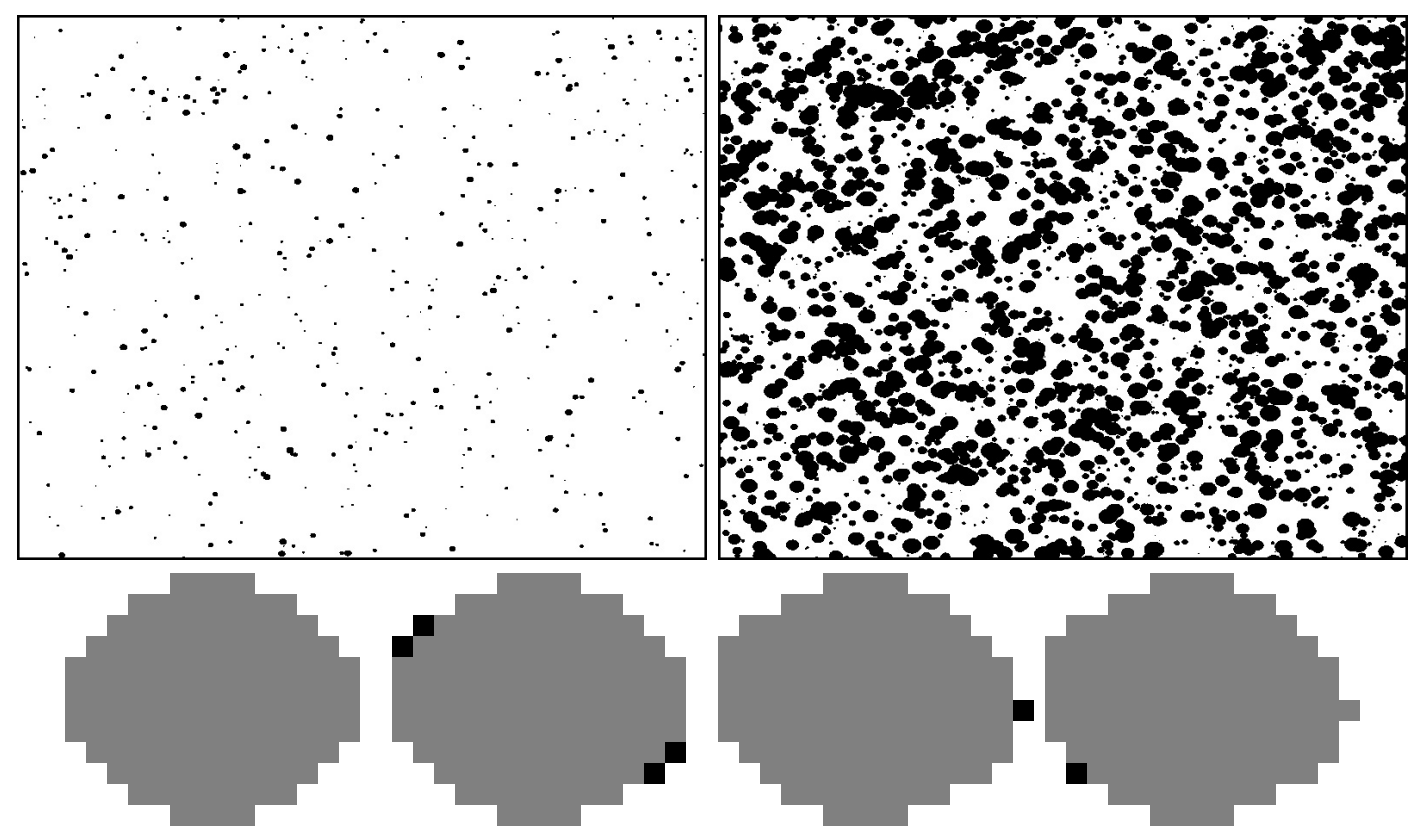

Figure 1. Two different time instants $(X$ and $Y)$ pictures of a simulated birth-and-growth process. The magnified pictures of the true growth used for the simulation, the computed $\widehat{G}_{W}^{2}$, $\widehat{G}_{W}^{1}$ and $\widehat{G}_{W \ominus \check{K}}^{1}$. Proposition 4.1 is satisfied since $\widehat{G}_{W \ominus \check{K}}^{1} \supseteq \widehat{G}_{W}^{1} \supseteq \widehat{G}_{W}^{2}$.

Proposition 4.2 (See [1]). Let $X, Y$ be a.s. regular closed. Let $G, B$ be two RaCS such that $Y=(X \oplus G) \cup B$, with $B$ a stationary ergodic RaCS independent on $G$ and $X$, and with $G$ a.s. regular closed. Then, for any $K \in \mathbb{F}_{k}$,

$$
\left|\widetilde{Q}_{B, W}(K)-Q_{B}(K)\right| \underset{W \uparrow \mathfrak{X}}{\underset{\longrightarrow}{\longrightarrow}} 0, \quad \text { a.s. }
$$

\section{A. Proofs of Propositions in Section 3.2}

Proof of Proposition 3.2. Let the $\sigma$-algebra generated by the above collection of sets be denoted by $\mathcal{P}^{\prime}$. We shall show $\mathcal{P}=\mathcal{P}^{\prime}$. Let $G$ be a left continuous process and let $\alpha=\left(T-t_{0}\right)$, consider for $n \in \mathbb{N}$

$$
G_{n}(\omega, t)=\left\{\begin{array}{lc}
G\left(\omega, t_{0}\right), & t=t_{0} \\
G\left(\omega, t_{0}+\frac{k \alpha}{2^{n}}\right), & \left(t_{0}+\frac{k \alpha}{2^{n}}\right)<t \leq\left(t_{0}+\frac{(k+1) \alpha}{2^{n}}\right) \\
\quad k \in\left\{0, \ldots,\left(2^{n}-1\right)\right\}^{n}
\end{array}\right.
$$

It is clear that $G_{n}$ is $\mathcal{P}^{\prime}$-measurable, since $G$ is adapted. As $G$ is left continuous, the above sequence of left-continuous processes converges pointwise (with respect to $\delta_{H}$ ) to $G$ when $n$ tends to infinity, so $G$ is $\mathcal{P}^{\prime}$ measurable, thus $\mathcal{P} \subseteq \mathcal{P}^{\prime}$. Conversely consider $A \times(s, t] \in \mathcal{P}^{\prime}$ with $(s, t] \subset\left[t_{0}, T\right]$ and $A \in \mathfrak{F}_{s}$. Let $b \in \mathfrak{X} \backslash\{0\}$ and $G$ be the process

$$
G(\omega, v)= \begin{cases}b, & v \in(s, t], \omega \in A \\ 0, & \text { otherwise }\end{cases}
$$

this function is adapted and left continuous, hence $\mathcal{P}^{\prime} \subseteq \mathcal{P}$.

In order to prove Proposition 3.3, let us recall the following properties for real processes. A real-valued process $X=\left\{X_{t}\right\}_{t \in\left[t_{0}, T\right]}$ is predictable with respect to filtration $\left\{\mathfrak{F}_{t}\right\}_{t \in \mathbb{R}_{+}}$, if it is measurable with respect to the 
predictable $\sigma$-algebra $\mathcal{P}_{\mathbb{R}}$, i.e. the $\sigma$-algebra generated by the collection of random sets $A \times\{0\}$ where $A \in \mathfrak{F}_{0}$ and $A \times(s, t]$ where $A \in \mathfrak{F}_{s}$.

Lemma A.1 (see ([11], Prop. 2.30, 2.32 and 2.41)). Let $X=\left\{X_{t}\right\}_{t \in\left[t_{0}, T\right]}$ be a predictable real-valued process, then $X$ is $\left(\mathfrak{F} \otimes \mathcal{B}_{\left[t_{0}, T\right]}, \mathcal{B}_{\mathbb{R}}\right)$-measurable. Further, for every $\omega \in \Omega$, the trajectory $X(\omega, \cdot):\left[t_{0}, T\right] \rightarrow \mathbb{R}$ is $\left(\mathcal{B}_{\left[t_{0}, T\right]}, \mathcal{B}_{\mathbb{R}}\right)$-measurable.

Lemma A.2. Let $x^{*}$ be an element of the unit ball $B_{1}^{*}$ of the dual space $\mathfrak{X}^{*}$, then $G \mapsto s\left(x^{*}, G\right)$ is a $\left(\mathcal{P}, \mathcal{B}_{\mathbb{R}}\right)$ measurable map.

Proof. By definition $s\left(x^{*}, G\right)=\sup \left\{x^{*}(g): g \in G\right\}$. Since $\mathfrak{X}$ is separable (A-0), there exists $\left\{g_{n}\right\}_{n \in \mathbb{N}} \subset G$ such that $G=\overline{\left\{g_{n}\right\}}$. Then, for every $x^{*} \in B_{1}^{*}$ we have

$$
s\left(x^{*}, G\right)=\sup _{g \in G} x^{*}(g)=\sup _{n \in \mathbb{N}} x^{*}\left(g_{n}\right)
$$

Since $x^{*}$ is an element of the dual space $\mathfrak{X}^{*}, x^{*}$ is a continuous map and then $s\left(x^{*}, \cdot\right)$ is measurable.

Proof of Proposition 3.3. Assumptions (A-3) and (A-4) imply that $G$ is non-empty and convex. Measurability and integrability properties are consequence of (A-6) and (A-5) respectively.

For the second part of proposition, we have to prove that $G_{a, b}$ is a non-empty, bounded convex RaCS.

First, we prove that $G_{a, b}$ is a measurable map. From the previous part, integral $G_{a, b}=\int_{a}^{b} G(\omega, \tau) \mathrm{d} \tau$ is well defined for all $\omega \in \Omega$. Assumption (A-3) implies $0 \in G_{a, b}(\omega) \neq \emptyset$ for every $\omega \in \Omega$. Hence, the domain of $G_{a, b}$ is the whole $\Omega$ for all $a, b \in\left[t_{0}, T\right]$

$$
D\left(G_{a, b}\right)=\left\{\omega \in \Omega: G_{a, b} \neq \emptyset\right\}=\Omega \in \mathfrak{F}
$$

Thus, by Proposition 2.2 and for a fixed couple $a, b \in\left[t_{0}, T\right], G_{a, b}$ is (weakly) measurable if and only if, for every $x \in \mathfrak{X}$, the map

$$
\omega \mapsto d\left(x, \int_{a}^{b} G(\omega, \tau) \mathrm{d} \tau\right)=\delta_{H}\left(x, \int_{a}^{b} G(\omega, \tau) \mathrm{d} \tau\right)
$$

is measurable. Equation (2.1) guarantees that (A.1) is measurable if and only if, for every $x \in \mathfrak{X}$, the map

$$
\omega \mapsto \sup _{x^{*} \in B_{1}^{*}}\left|s\left(x^{*}, x\right)-s\left(x^{*}, \int_{a}^{b} G(\omega, \tau) \mathrm{d} \tau\right)\right|
$$

is measurable. The above expression can be computed on a countable family dense in $B_{1}^{*}$ (note that such family exists since $\mathfrak{X}^{*}$ is assumed separable $(\mathrm{A}-0)$ )

$$
\omega \mapsto \sup _{n \in \mathbb{N}}\left|s\left(x_{i}^{*}, x\right)-s\left(x_{i}^{*}, \int_{a}^{b} G(\omega, \tau) \mathrm{d} \tau\right)\right| .
$$

It can be proved ([25], Thm. 2.1.12, p. 46) that

$$
s\left(x^{*}, \int_{a}^{b} G(\omega, \tau) \mathrm{d} \tau\right)=\int_{a}^{b} s\left(x^{*}, G(\omega, \tau)\right) \mathrm{d} \tau, \quad \forall x^{*} \in B_{1}^{*}
$$

and therefore, since $s\left(x_{i}^{*}, x\right)$ is a constant, $G_{a, b}$ is measurable if, for every $x^{*} \in\left\{x_{i}^{*}\right\}_{i \in \mathbb{N}}$, the following map

$$
\begin{aligned}
(\Omega, \mathfrak{F}) & \rightarrow\left(\mathbb{R}, \mathcal{B}_{\mathbb{R}}\right) \\
\omega & \mapsto \int_{a}^{b} s\left(x^{*}, G(\omega, \tau)\right) \mathrm{d} \tau
\end{aligned}
$$


is measurable. Note that $s\left(x^{*}, G(\cdot, \cdot)\right)$, as a map from $\Omega \times\left[t_{0}, T\right]$ to $\mathbb{R}$, is predictable since it is the composition of a predictable map (A-6) with a measurable one (see Lem. A.2):

$$
\begin{array}{ccccc}
s\left(x^{*}, G(\cdot, \cdot)\right):\left(\Omega \times\left[t_{0}, T\right], \mathcal{P}\right) & \rightarrow & \left(\mathbb{F}^{\prime}, \sigma_{f}\right) & \rightarrow & \left(\mathbb{R}, \mathcal{B}_{\mathbb{R}}\right) \\
(\omega, t) & \mapsto & G(\omega, t) & \mapsto s\left(x^{*}, G(\omega, t)\right)
\end{array}
$$

thus, by Lemma A.1, it is a $\mathcal{P}$-measurable map and hence (A.2) is a measurable map.

In view of the first part, it remains to prove that $G_{a, b}$ is a bounded convex set for a.e. $\omega \in \Omega$. From the first part of proof and since $\mathfrak{X}$ is reflexive (A-0), we get that $G_{a, b}$ is closed (see ([25], Thm. 2.2.3)). Further, $G_{a, b}$ is also convex (see ([25], Thm. 2.1.5 and Cor. 2.1.6)).

To conclude the proof, it is sufficient to show that $G_{a, b}$ is included in a bounded set:

$$
\begin{aligned}
\int_{a}^{b} G(\omega, \tau) \mathrm{d} \tau & =\left\{\int_{a}^{b} g(\omega, \tau) \mathrm{d} \tau: g(\omega, \cdot) \in G(\omega, \cdot) \subseteq K\right\} \\
& \subseteq\left\{\int_{a}^{b} k \mathrm{~d} \tau: k \in K\right\}=\{(b-a) k: k \in K\}=(b-a) K
\end{aligned}
$$

Proof of Proposition 3.4. For every $i \in\{0, \ldots, n\}, \int_{t_{i-1}}^{t} G(\tau) \mathrm{d} \tau$ is a RaCS (Proposition 3.3). Thus, measurability Assumption (A-1) on $B$ guarantees that, for every $t_{i} \in \Pi, B_{t_{i}}, \Delta B_{t_{i}},\left(\Delta B_{t_{i}} \oplus \int_{t_{i}}^{t} G(\tau) \mathrm{d} \tau\right)$, and hence $s_{\Pi}$ and $S_{\Pi}$ are RaCS.

Lemma A.3. Let $X \in L^{1}\left[I, \mathfrak{F}, \mu_{\lambda} ; \mathbb{F}^{\prime}\right]$, where $I$ is a bounded interval of $\mathbb{R}$, such that $0 \in X \mu_{\lambda}$-almost everywhere on $I$ and let $I_{1}, I_{2}$ be two other intervals of $\mathbb{R}$ with $I_{1} \subset I_{2} \subset I$. Then

$$
\int_{I_{1}} X(\tau) \mathrm{d} \tau \subseteq \int_{I_{2}} X(\tau) \mathrm{d} \tau
$$

Proof. Let $y \in\left(\int_{I_{1}} X(\tau) \mathrm{d} \tau\right)$, then there exists $x \in S_{X}$, for which $y=\left(\int_{I_{1}} x(\tau) \mathrm{d} \tau\right)$. Let us define on $I_{2}\left(\supset I_{1}\right)$

$$
x^{\prime}(\tau)= \begin{cases}x(\tau), & \tau \in I_{1} \\ 0, & \tau \in I_{2} \backslash I_{1}\end{cases}
$$

then $x^{\prime} \in S_{X}$ and $y=\left(\int_{I_{2}} x^{\prime}(\tau) \mathrm{d} \tau\right) \in\left(\int_{I_{2}} X(\tau) \mathrm{d} \tau\right)$.

Proof of Proposition 3.5. The thesis is a consequence of Lemma A.3 and Minkowski addition properties, in fact $\left(\int_{t_{i-1}}^{t} G(\tau) \mathrm{d} \tau\right) \subseteq\left(\int_{t_{i}}^{t} G(\tau) \mathrm{d} \tau\right)$ implies $s_{\Pi} \subseteq S_{\Pi}$.

Proof of Proposition 3.6. Let $\Pi^{\prime}$ be a refinement of partition $\Pi$ of $\left[t_{0}, t\right]$, i.e. $\Pi \subset \Pi^{\prime}$. We prove that $s_{\Pi} \subseteq s_{\Pi^{\prime}}$ ( $S_{\Pi^{\prime}} \subseteq S_{\Pi}$ is analogous). It is sufficient to show the thesis only for $\Pi^{\prime}=\Pi \cup\{\bar{t}\}$ where $\Pi=\left\{t_{0}, \ldots, t_{n}\right\}$ with $t_{0}<\ldots<t_{n}=t$ and $\bar{t} \in\left(t_{0}, t\right)$. Let $i \in\{0, \ldots,(n-1)\}$ be such that $t_{i} \leq \bar{t} \leq t_{i+1}$ then

$$
s_{\Pi}=\left(B_{t_{0}} \oplus \int_{t_{0}}^{t} G(\tau) \mathrm{d} \tau\right) \cup \bigcup_{\substack{j=1 \\ j \neq i+1}}^{n}\left(\Delta B_{t_{j}} \oplus \int_{t_{j}}^{t} G(\tau) \mathrm{d} \tau\right) \cup\left[\left(B_{t_{i+1}} \backslash B_{t_{i}}^{o}\right) \oplus \int_{t_{i+1}}^{t} G(\tau) \mathrm{d} \tau\right]
$$


and

$$
\begin{aligned}
& s_{\Pi^{\prime}}=\left(B_{t_{0}} \oplus \int_{t_{0}}^{t} G(\tau) \mathrm{d} \tau\right) \cup \bigcup_{\substack{j=1 \\
j \neq i+1}}^{n}\left(\Delta B_{t_{j}} \oplus \int_{t_{j}}^{t} G(\tau) \mathrm{d} \tau\right) \\
& \cup\left[\left(B_{\bar{t}} \backslash B_{t_{i}}^{o}\right) \oplus \int_{\bar{t}}^{t} G(\tau) \mathrm{d} \tau\right] \cup\left[\left(B_{t_{i+1}} \backslash B_{\bar{t}}^{o}\right) \oplus \int_{t_{i+1}}^{t} G(\tau) \mathrm{d} \tau\right]
\end{aligned}
$$

Definitely, in order to prove that $s_{\Pi} \subseteq s_{\Pi^{\prime}}$ we have to prove that

$$
\left[\left(B_{t_{i+1}} \backslash B_{t_{i}}^{o}\right) \oplus \int_{t_{i+1}}^{t} G(\tau) \mathrm{d} \tau\right] \subseteq\left[\left(B_{\bar{t}} \backslash B_{t_{i}}^{o}\right) \oplus \int_{\bar{t}}^{t} G(\tau) \mathrm{d} \tau\right] \cup\left[\left(B_{t_{i+1}} \backslash B_{\bar{t}}^{o}\right) \oplus \int_{t_{i+1}}^{t} G(\tau) \mathrm{d} \tau\right]
$$

This inclusion is a consequence of $\left(\int_{t_{i+1}}^{t} G(\tau) \mathrm{d} \tau\right) \subseteq\left(\int_{\frac{t}{t}}^{t} G(\tau) \mathrm{d} \tau\right)$ (Lem. A.3) and of the Minkowski sum distribution property.

Proof of Proposition 3.7. Let $\Pi_{j}=\left(t_{i}\right)_{i=0}^{n}$ be the $j$-partition of the refinement sequence $\left\{\Pi_{j}\right\}_{j \in \mathbb{N}}$, then

$$
\delta_{H}\left(s_{\Pi_{j}}, S_{\Pi_{j}}\right)=\max \left\{\sup _{x \in s_{\Pi_{j}}} \mathrm{~d}\left(x, S_{\Pi_{j}}\right), \sup _{y \in S_{\Pi_{j}}} \mathrm{~d}\left(y, s_{\Pi_{j}}\right)\right\}
$$

where $\mathrm{d}\left(x, S_{\Pi_{j}}\right)=\inf _{y \in S_{\Pi_{j}}}\|x-y\|_{\mathfrak{X}}$. By Proposition $3.5, s_{\Pi_{j}} \subseteq S_{\Pi_{j}}$ then

$$
\sup _{x \in s_{\Pi_{j}}} \mathrm{~d}\left(x, S_{\Pi_{j}}\right)=0
$$

and hence we have to prove that, whenever $j \rightarrow \infty\left(\right.$ i.e. $\left.\left|\Pi_{j}\right| \rightarrow 0\right)$,

$$
\delta_{H}\left(s_{\Pi_{j}}, S_{\Pi_{j}}\right)=\sup _{y \in S_{\Pi_{j}}} \mathrm{~d}\left(y, s_{\Pi_{j}}\right)=\sup _{y \in S_{\Pi_{j}}} \inf _{x \in s_{\Pi_{j}}}\|x-y\|_{\mathfrak{X}} \longrightarrow 0 .
$$

For every $\omega \in \Omega$, let $y$ be any element of $S_{\Pi_{j}}(\omega)$, then we distinguish two cases:

(1) if $y \in\left(B_{t_{0}}(\omega) \oplus \int_{t_{0}}^{t} G(\omega, \tau) \mathrm{d} \tau\right)$, then it is also an element of $s_{\Pi_{j}}(\omega)$, and hence $d\left(s_{\Pi_{j}}(\omega), y\right)=0$.

(2) if $y \notin\left(B_{t_{0}}(\omega) \oplus \int_{t_{0}}^{t} G(\omega, \tau) \mathrm{d} \tau\right)$, then there exist $j \in\{1, \ldots, n\}$ such that

$$
y \in\left(\Delta B_{t_{j}}(\omega) \oplus \int_{t_{j-1}}^{t} G(\omega, \tau) \mathrm{d} \tau\right) .
$$

By definition of $\oplus$, for every $\omega \in \Omega$, there exist

$$
\left\{y_{m}\right\}_{m \in \mathbb{N}} \subseteq\left(\Delta B_{t_{j}}(\omega)+\int_{t_{j-1}}^{t} G(\omega, \tau) \mathrm{d} \tau\right),
$$

such that $\lim _{m \rightarrow \infty} y_{m}=y$. Then, for every $\omega \in \Omega$, there exist $h_{m} \in \Delta B_{t_{j}}(\omega)$ and $g_{m} \in\left(\int_{t_{j-1}}^{t} G(\omega, \tau) \mathrm{d} \tau\right)$ such that $y_{m}=\left(h_{m}+g_{m}\right)$ and hence

$$
y=\lim _{m \rightarrow \infty}\left(h_{m}+g_{m}\right)=\lim _{m \rightarrow \infty} y_{m}
$$


where the convergence is in the Banach norm, then let $\bar{m} \in \mathbb{N}$ be such that $\left\|y-y_{m}\right\|_{\mathfrak{X}}<\left|\Pi_{j}\right|$, for every $m>\bar{m}$.

Note that, for every $\omega \in \Omega$ and $m \in \mathbb{N}$, by Aumann integral definition, there exists a selection $\widehat{g_{m}}(\cdot)$ of $G(\omega, \cdot)$ (i.e. $\widehat{g_{m}}(t) \in G(\omega, t) \mu_{\lambda^{-}}$a.e. $)$such that

$$
g_{m}=\int_{t_{j-1}}^{t} \widehat{g_{m}}(\tau) \mathrm{d} \tau \quad \text { and } \quad y_{m}=h_{m}+\int_{t_{j-1}}^{t} \widehat{g_{m}}(\tau) \mathrm{d} \tau .
$$

For every $\omega \in \Omega$, let us consider

$$
x_{m}=h_{m}+\int_{t_{j}}^{t} \widehat{g_{m}}(\tau) \mathrm{d} \tau
$$

then $x_{m} \in s_{\Pi_{j}}(\omega)$ for all $m \in \mathbb{N}$. Moreover, the following chain of inequalities hold, for all $m>\bar{m}$ and $\omega \in \Omega$,

$$
\begin{aligned}
\inf _{x^{\prime} \in s_{\Pi_{j}}}\left\|x^{\prime}-y\right\|_{\mathfrak{X}} & \leq\left\|x_{m}-y\right\|_{\mathfrak{X}} \leq\left\|x_{m}-y_{m}\right\|_{\mathfrak{X}}+\left\|y_{m}-y\right\|_{\mathfrak{X}} \\
& \leq\left\|\int_{t_{j-1}}^{t_{j}} \widehat{g_{m}}(\tau) \mathrm{d} \tau\right\|_{\mathfrak{X}}+\left|\Pi_{j}\right| \leq \int_{t_{j-1}}^{t_{j}}\left\|\widehat{g_{m}}(\tau)\right\|_{\mathfrak{X}} \mathrm{d} \tau+\left|\Pi_{j}\right| \\
& \leq \int_{t_{j-1}}^{t_{j}} \delta_{H}(G(\tau),\{0\}) \mathrm{d} \tau+\left|\Pi_{j}\right| \leq\left|t_{j}-t_{j-1}\right| \delta_{H}(K,\{0\})+\left|\Pi_{j}\right| \\
& \leq\left|\Pi_{j}\right|\left(\delta_{H}(K,\{0\})+1\right) \stackrel{j \rightarrow \infty}{\longrightarrow} 0
\end{aligned}
$$

since $\delta_{H}(K,\{0\})$ is a positive constant. By the arbitrariness of $y \in S_{\Pi_{j}}(\omega)$ we obtain the thesis.

Proof of Proposition 3.8. Let $\Pi_{j}$ and $\Pi_{l}^{\prime}$ be two partitions of the two distinct refinement sequences $\left\{\Pi_{j}\right\}_{j \in \mathbb{N}}$ and $\left\{\Pi_{l}^{\prime}\right\}_{l \in \mathbb{N}}$ of $\left[t_{0}, t\right]$. Let $\Pi^{\prime \prime}=\Pi_{j} \cup \Pi_{l}^{\prime}$ be the refinement of both $\Pi_{j}$ and $\Pi_{l}^{\prime}$. Then Proposition 3.6 and Proposition 3.5 imply that $s_{\Pi_{j}} \subseteq s_{\Pi^{\prime \prime}} \subseteq S_{\Pi^{\prime \prime}} \subseteq S_{\Pi_{l}^{\prime}}$. Therefore $s_{\Pi_{j}} \subseteq S_{\Pi_{l}^{\prime}}$ for every $j, l \in \mathbb{N}$. Then

$$
\overline{\left(\bigcup_{j \in \mathbb{N}} s_{\Pi_{j}}\right)} \subseteq \bigcap_{l \in \mathbb{N}} S_{\Pi_{l}^{\prime}}
$$

Analogously

$$
\overline{\left(\bigcup_{l \in \mathbb{N}} s_{\Pi_{l}^{\prime}}\right)} \subseteq \bigcap_{j \in \mathbb{N}} S_{\Pi_{j}} .
$$

Proposition 3.7 concludes the proof.

In order to prove Theorem 3.11, let us consider the following lemma that shows how $s_{\Pi}(t)$ and $S_{\Pi}(t)$ are not decreasing with respect to time $t$.

Lemma A.4. Let $s, t \in\left[t_{0}, T\right]$ with $t_{0}<s<t$ and let $\Pi$ be a partition of $\left[t_{0}, t\right]$. Then

$$
s_{\Pi}(s) \subseteq s_{\Pi}(t) \quad \text { and } \quad S_{\Pi}(s) \subseteq S_{\Pi}(t) .
$$


Proof. The proofs of the two inclusions are similar. Let us prove that $s_{\Pi}(s) \subseteq s_{\Pi}(t)$. By Lemma A.3, we have that

$$
\begin{aligned}
s_{\Pi}(s) & =\left(B_{t_{0}} \oplus \int_{t_{0}}^{s} G(\tau) \mathrm{d} \tau\right) \cup \bigcup_{\substack{t^{\prime} \in \Pi \\
t^{\prime} \leq s}}\left(\Delta B_{t^{\prime}} \oplus \int_{t^{\prime}}^{s} G(\tau) \mathrm{d} \tau\right) \\
& \subseteq\left(B_{t_{0}} \oplus \int_{t_{0}}^{t} G(\tau) \mathrm{d} \tau\right) \cup \bigcup_{\substack{t^{\prime} \in \Pi \\
t^{\prime} \leq s}}\left(\Delta B_{t^{\prime}} \oplus \int_{t^{\prime}}^{t} G(\tau) \mathrm{d} \tau\right) \\
& \subseteq\left(B_{t_{0}} \oplus \int_{t_{0}}^{t} G(\tau) \mathrm{d} \tau\right) \cup \bigcup_{t^{\prime} \in \Pi}\left(\Delta B_{t^{\prime}} \oplus \int_{t^{\prime}}^{t} G(\tau) \mathrm{d} \tau\right)
\end{aligned}
$$

i.e. $s_{\Pi}(s) \subseteq s_{\Pi}(t)$.

Proof of Theorem 3.11. For every $s, t \in\left[t_{0}, T\right]$ with $s<t$, let $\left\{\Pi_{i}\right\}_{i \in \mathbb{N}}$ be a refinement sequence of $\left[t_{0}, t\right]$, such that $s \in \Pi_{1}$ (and hence $s \in \Pi_{i}$ for every $i \in \mathbb{N}$ ). Then, by Lemma A.4, $S_{\Pi_{i}}(s) \subseteq S_{\Pi_{i}}(t)$. Now, as $i$ tends to infinity, we obtain

$$
\Theta_{s}=\bigcap_{i \rightarrow \infty} S_{\Pi_{i}}(s) \subseteq \bigcap_{i \rightarrow \infty} S_{\Pi_{i}}(t)=\Theta_{t} .
$$

For the second part, note that Proposition 3.3 still holds replacing $\mathfrak{F}_{t}$ instead of $\mathfrak{F}$, so that for every $s \in\left[t_{0}, T\right]$, the family $\left\{\int_{s}^{t} G(\omega, \tau) \mathrm{d} \tau\right\}_{t \in[s, T]}$ is an adapted process to the filtration $\left\{\mathfrak{F}_{t}\right\}_{t \in\left[t_{0}, T\right]}$. This fact together with Assumption (A-1) guarantees that $\left\{S_{\Pi}\right\}_{t \in[s, T]}$ is adapted for every partition $\Pi$ of $[s, T]$ and hence $\Theta$ is adapted too.

\section{REFERENCES}

[1] G. Aletti, E.G. Bongiorno and V. Capasso, Statistical aspects of fuzzy monotone set-valued stochastic processes. application to birth-and-growth processes. Fuzzy Set. Syst. 160 (2009) 3140-3151.

[2] G. Aletti and D. Saada, Survival analysis in Johnson-Mehl tessellation. Stat. Infer. Stoch. Process. 11 (2008) 55-76.

[3] D. Aquilano, V. Capasso, A. Micheletti, S. Patti, L. Pizzocchero and M. Rubbo, A birth and growth model for kinetic-driven crystallization processes, part i: Modeling. Nonlinear Anal. Real World Appl. 10 (2009) 71-92.

[4] J. Aubin and H. Frankowska, Set-valued Analysis. Birkhäuser, Boston Inc. (1990).

[5] G. Barles, H.M. Soner and P.E. Souganidiss, Front propagation and phase field theory. SIAM J. Control Optim. 31 (1993) 439-469.

[6] M. Burger, Growth fronts of first-order Hamilton-Jacobi equations. SFB Report 02-8, University Linz, Linz, Austria (2002).

[7] M. Burger, V. Capasso and A. Micheletti, An extension of the Kolmogorov-Avrami formula to inhomogeneous birth-and-growth processes, in Math Everywhere. G. Aletti et al. Eds., Springer, Berlin (2007) 63-76.

[8] M. Burger, V. Capasso and L. Pizzocchero, Mesoscale averaging of nucleation and growth models. Multiscale Model. Simul. 5 (2006) 564-592 (electronic).

[9] V. Capasso (Ed.) Mathematical Modelling for Polymer Processing. Polymerization, Crystallization, Manufacturing. Mathematics in Industry 2, Springer-Verlag, Berlin (2003).

[10] V. Capasso, On the stochastic geometry of growth, in Morphogenesis and Pattern Formation in Biological Systems. T. Sekimura, et al. Eds., Springer, Tokyo (2003) 45-58.

[11] V. Capasso and D. Bakstein, An Introduction to Continuous-Time Stochastic Processes. Modeling and Simulation in Science, Engineering and Technology, Birkhäuser Boston Inc. (2005).

[12] V. Capasso and E. Villa, Survival functions and contact distribution functions for inhomogeneous, stochastic geometric marked point processes. Stoch. Anal. Appl. 23 (2005) 79-96.

[13] C. Castaing and M. Valadier, Convex Analysis and Measurable Multifunctions. Lecture Notes Math. 580, Springer-Verlag, Berlin (1977).

[14] S.N. Chiu, Johnson-Mehl tessellations: asymptotics and inferences, in Probability, finance and insurance. World Sci. Publ., River Edge, NJ (2004) 136-149.

[15] S.N. Chiu, I.S. Molchanov and M.P. Quine, Maximum likelihood estimation for germination-growth processes with application to neurotransmitters data. J. Stat. Comput. Simul. 73 (2003) 725-732. 
[16] N. Cressie, Modeling growth with random sets. In Spatial Statistics and Imaging (Brunswick, ME, 1988). IMS Lecture Notes Monogr. Ser. 20, Inst. Math. Statist., Hayward, CA (1991) 31-45.

[17] D.J. Daley and D. Vere-Jones, An Introduction to the Theory of Point Processes. Probability and its Applications, I, 2nd edition, Springer-Verlag, New York (2003).

[18] N. Dunford and J.T. Schwartz, Linear Operators. Part I. Wiley Classics Library, John Wiley \& Sons Inc., New York (1988).

[19] T. Erhardsson, Refined distributional approximations for the uncovered set in the Johnson-Mehl model. Stoch. Proc. Appl. 96 (2001) 243-259.

[20] H.J. Frost and C.V. Thompson, The effect of nucleation conditions on the topology and geometry of two-dimensional grain structures. Acta Metallurgica 35 (1987) 529-540.

[21] E. Giné, M.G. Hahn and J. Zinn, Limit theorems for random sets: an application of probability in Banach space results. In Probability in Banach Spaces, IV (Oberwolfach, 1982). Lecture Notes Math. 990, Springer, Berlin (1983) $112-135$.

[22] J. Herrick, S. Jun, J. Bechhoefer and A. Bensimon, Kinetic model of DNA replication in eukaryotic organisms. J. Mol. Biol. 320 (2002) 741-750.

[23] F. Hiai and H. Umegaki, Integrals, conditional expectations, and martingales of multivalued functions. J. Multivariate Anal. 7 (1977) 149-182.

[24] C.J. Himmelberg, Measurable relations. Fund. Math. 87 (1975) 53-72.

[25] S. Li, Y. Ogura and V. Kreinovich, Limit Theorems and Applications of Set-Valued and Fuzzy Set-Valued Random Variables. Kluwer Academic Publishers Group, Dordrecht (2002).

[26] G. Matheron, Random Sets and Integral Geometry, John Wiley \& Sons, New York-London-Sydney (1975).

[27] A. Micheletti, S. Patti and E. Villa, Crystal growth simulations: a new mathematical model based on the Minkowski sum of sets, in Industry Days 2003-2004 The MIRIAM Project 2, D. Aquilano et al. Eds., Esculapio, Bologna (2005) 130-140.

[28] I.S. Molchanov, Statistics of the Boolean Model for Practitioners and Mathematicians. Wiley, Chichester (1997).

[29] I.S. Molchanov and S.N. Chiu, Smoothing techniques and estimation methods for nonstationary Boolean models with applications to coverage processes. Biometrika 87 (2000) 265-283.

[30] J. Møller, Random Johnson-Mehl tessellations. Adv. Appl. Prob. 24 (1992) 814-844.

[31] J. Møller, Generation of Johnson-Mehl crystals and comparative analysis of models for random nucleation. Adv. Appl. Prob. 27 (1995) 367-383.

[32] J. Møller and M. Sørensen, Statistical analysis of a spatial birth-and-death process model with a view to modelling linear dune fields. Scand. J. Stat. 21 (1994) 1-19.

[33] H. Rådström, An embedding theorem for spaces of convex sets. Proc. Am. Math. Soc. 3 (1952) 165-169.

[34] J. Serra, Image Analysis and Mathematical Morphology. Academic Press Inc., London (1984).

[35] L. Shoumei and R. Aihong, Representation theorems, set-valued and fuzzy set-valued Ito integral. Fuzzy Set. Syst. 158 (2007) 949-962.

[36] D. Stoyan, W.S. Kendall and J. Mecke, Stochastic Geometry and its Applications. 2nd edition, John Wiley \& Sons Ltd., Chichester (1995). 